\title{
Analysis of microbial differences in amniotic fluid between advanced and normal age pregnant women
}

\author{
Ya Wang ${ }^{1 \dagger}$, Chunyu Luo ${ }^{1 \dagger}$, Yiwei Cheng ${ }^{2}$, Li Li ${ }^{1}$, Dong Liang ${ }^{1}$, Ping Hu ${ }^{1 *}$ and Zhengfeng $\mathrm{Xu}^{1^{*}}$ (D)
}

Keywords: Amniotic fluid, Microorganisms, 16S rRNA gene sequencing, Proteomics

\section{To the Editor}

Amniotic fluid (AF) has been considered sterile for nearly a century because no microorganisms were identified by traditional culture methods [1]. However, this opinion paradigm has been challenged by recent studies based on culture-independent sequencing techniques [2-4] and AF proteomics [5]. Recently, a conclusion that no microorganisms were present in the mid-trimester AF of healthy pregnancies was reached using culture-independent sequencing techniques [6] and seemed to settle the argument [7]. However, it could not explain why nonhuman proteins were identified in normal human $\mathrm{AF}$ supernatants [8] and why microbial exposure primes fetal immune cells in fetal tissues during fetal development [9].

Intra-amniotic infection caused by microbial invasion of the amniotic cavity (MIAC) was associated with adverse pregnancy outcomes, when the bacteria were at high concentrations [10]. This may be a reason why people think that AF is sterile and that bacteria in AF are abnormal because of their negative effects.

Meanwhile, we could identify bacterial proteins in the AF proteomics database that were consistent with the results of $16 \mathrm{~S}$ ribosomal RNA (rRNA) gene sequencing. Nine amniotic fluid samples were collected from 9 pregnant women and the pregnancy outcomes of the

\footnotetext{
*Correspondence: njfybjyhuping@163.com; zhengfeng_xu_nj@163.com †Ya Wang and Chunyu Luo contributed equally

${ }^{1}$ Department of Prenatal Diagnosis, Obstetrics and Gynecology Hospital

Affiliated to Nanjing Medical University, Nanjing Maternity and Child Health Care Hospital, Nanjing 210004, China

Full list of author information is available at the end of the article
}

participants were followed. No bacteria were found by cultivation, but a sparse microbial presence was found by proteomics analysis and $16 \mathrm{~S}$ rRNA gene sequencing approach. The 148 microbes found in the human $\mathrm{AF}$ proteomics database were consistent with the microbes found in the 16S rRNA gene sequencing database. The species composition and the structure of communities in the normal age ( $<35$ years old) and advanced maternal age (AMA) (> 35 years old) pregnancies differed significantly. However, all of the newborns were healthy and had no allergic reactions.

Proteomics analysis has identified nearly 2000 proteins in AF during the past 20 years [11], including many nonhuman proteins [8]. In a recent study, 7 normal AF samples were used to generate human AF proteomes, which were divided into 4 groups: original proteins, bound proteins, flow-through proteins, and iTRAQ-labeled individual/mixed digested peptides [11]. We reanalyzed the human $\mathrm{AF}$ protein database and found that a part of the non-human proteins were derived from microorganisms. A total of 148 microbial-associated proteins in the normal human AF proteome, which could potentially play important roles in cellular/metabolic processes and binding/catalytic activity (Additional file 1). Notably, all of the microbial-associated proteins were present at a low level in human AF. These data suggest that there may be low concentrations of microorganisms in normal human AF samples.

To test our hypothesis, we conducted a study to investigate the presence of microorganisms in the mid-trimester AF of 9 women [ 4 normal age [normal] and 5 advanced

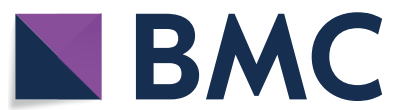

(c) The Author(s) 2021. Open Access This article is licensed under a Creative Commons Attribution 4.0 International License, which permits use, sharing, adaptation, distribution and reproduction in any medium or format, as long as you give appropriate credit to the original author(s) and the source, provide a link to the Creative Commons licence, and indicate if changes were made. The images or other third party material in this article are included in the article's Creative Commons licence, unless indicated otherwise in a credit line to the material. If material is not included in the article's Creative Commons licence and your intended use is not permitted by statutory regulation or exceeds the permitted use, you will need to obtain permission directly from the copyright holder. To view a copy of this licence, visit http://creativecommons.org/licenses/by/4.0/. The Creative Commons Public Domain Dedication waiver (http://creativeco mmons.org/publicdomain/zero/1.0/) applies to the data made available in this article, unless otherwise stated in a credit line to the data. 
maternal age [AMA]; advanced maternal age is generally defined as age above 35 years at the time of delivery [12, 13]) by cultivation and $16 \mathrm{~S}$ rRNA gene sequencing, and to follow their pregnancy outcomes. If the AMA group differed from the normal group in the microbial-associated 16S rRNA genes, it could reflect an endogenous microbial difference between the two groups and support the hypothesis that the AF is not sterile; otherwise, the microorganisms could be generated by exogenous contamination, which could not rule out the possibility that $\mathrm{AF}$ is sterile.

The main findings were: (1) B-ultrasonography and karyotype findings of the fetuses were normal and no bacteria were found by cultivation; (2) all 148 microbialassociated proteins in the normal human AF proteome were found in the 16S rRNA gene sequencing database, in which Bacillus, Mycobacterium, and Pseudomonas accounted for $\sim 20 \%$; (3) the bacterial richness of the AF samples showed no significant difference between the AMA and normal groups (Chao1 index, Welch's $t$ test, $P=0.540$; Additional file 2); (4) a significant difference in the species composition and structure of communities in the AF samples was found between the normal and AMA groups (Fig. 1A, B, Weighted_unifrac, OUT, Welch's $t$ test, $P=0.017$ ); (5) the newborns were healthy and had no allergic reactions up to 90 days (Table 1). Collectively, these data suggest that the normal AF is not sterile and that the species composition and structure of communities change in the AMA group, although the bacterial richness may be similar and have no effect on the babies' health.

The differences between the AF samples from the normal and AMA groups with regard to bacterial species composition and structure of communities were as follows. Five bacterial operational taxonomic units (OTUs) were increased in the AMA AF samples: Lactobacillus helveticus, Pediococcus acidilacticii, Pasteurella multocida, Bacillus indicus, and Bacteroides vulgatus. We used the LEfSe (Linear discriminant analysis Effect Size) method to identify bacterial OTUs that were likely to explain most of the differences between the normal and AMA AF samples. The bacterial orders of OTUs differed between the two groups. Sphingomonadales were more abundant in the normal group, while Lactobacillales were more abundant in the AMA group (Fig. 1C, LDA scores $>4$, Additional file 3). The differences in OTUs mainly spanned two Orders, with the Families Lactobacillaceae (primarily Lactobacillus helveticus) and Sphingomonadaceae accounting for the majority of the differences. Collectively, there may be a low concentration of microorganisms in the normal human AF samples and significant differences between the normal and AMA AF samples.
In the present study, we found that microbial-associated proteins and 16S rRNA genes could be identified in human AF at a low concentration. Furthermore, the species composition and structure of communities differed significantly between the normal and AMA AF samples. Thus, we conclude that the microbialassociated $16 \mathrm{~S}$ rRNA gene in human AF is real, rather than occurring through microbial contamination, and that the bacteria in AF differ between normal age and AMA pregnancies. We already know that AF neutrophils can phagocytize bacteria during intra-amniotic infection [14], but we do not know the function of bacteria in normal AF. Two possible hypotheses were proposed based on our results. First, we suggest that the AF is not sterile, but the level of microbiota may be very low and under the mother's immune system control. Therefore, the microbial communities may be related to the establishment of fetal immune function. Immunoglobulins from the mother may help to control the number or activity of the bacteria, to precisely control and activate the fetal immune system, given that recent studies demonstrated the presence of microbes or microbial DNA in the placenta, amniotic fluid [15], and meconium. Furthermore, Florent et al. [9] found live microbes in human fetuses such as Lactobacillus, and suggested that the selective presence of live microbes in fetal organs may have broader implications toward the establishment of immune competency and priming before birth, with the microbial exposure priming fetal immune cells during early human development. Second, we suggest that the bacterial 16S rRNA gene may come from the mother, because Rodriguez and colleagues proved that bacteria are transferred to the fetus from the mother by testing the meconium of healthy babies [16]. In addition, circulating cell-free DNA fragments are able to transfer between the fetus and the mother [17], and the bacterial 16S rRNA gene can be successfully detected in cell-free plasma DNA [18]. In this way, the bacterial $16 \mathrm{~S}$ rRNA gene could possibly enter the AF through the umbilical cord, and this would explain why we were able to identify the $16 \mathrm{~S}$ rRNA gene and peptides, but could not cultivate the bacteria.

\section{Materials and methods}

\section{AF sample collection and preparation}

Human amniotic fluid samples $(\sim 10 \mathrm{ml})$ were obtained by amniocentesis from women at 18-22 weeks of gestation who were undergoing prenatal diagnosis due to AMA or noninvasive prenatal testing for high-risk pregnancy after receiving written informed consent. The samples were collected in an operating room with the help of 
A

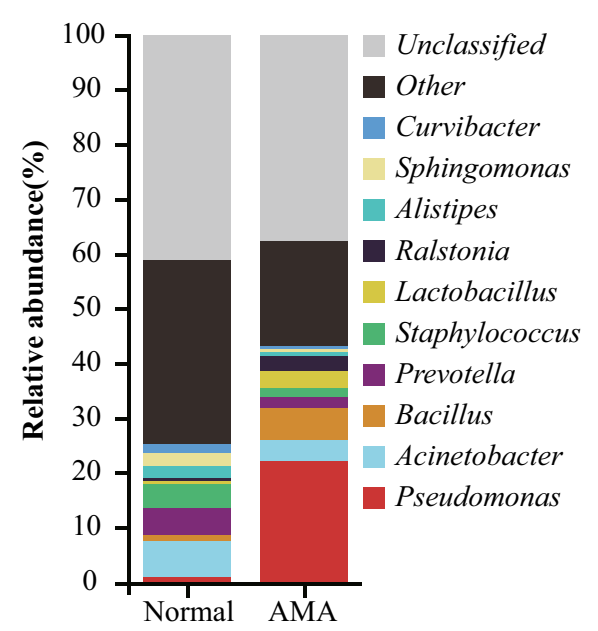

C

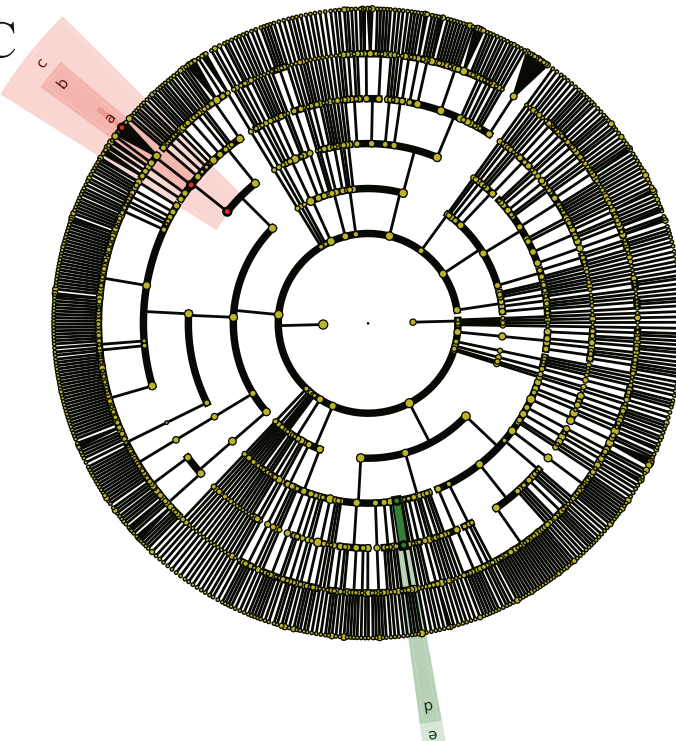

$\mathrm{B}$

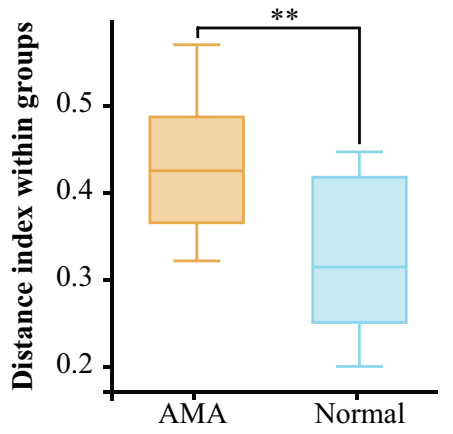

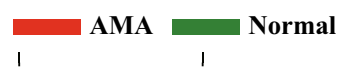

a: Sphingomonadaceae e: Sphingomonadales

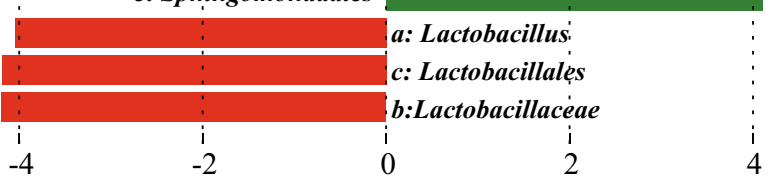

LDA Score $(\log 10)$

Fig. 1 Differences between the normal and AMA AF samples in species composition and structure of communities. A Detected compositions and relative abundances of bacterial communities according to $16 \mathrm{~S}$ rRNA gene sequencing. The normal age (left) and AMA (right) bacterial relative abundances of the top 10 bacterial communities were labeled with different colors. Unclassified: unclassified bacterial communities; others: other bacterial communities. B Comparison of distance indexes indicating species composition and structure of communities between the normal and AMA groups. (Weighted_unifrac, Welch's $t$ test, $P=0.017$ ). C Circular cladogram indicating the polygenetic distribution of the bacterial lineages in the AF samples from the normal and AMA groups as determined by the linear discriminant analysis (LDA) effect size (LEfSe) (right) and comparison of the LDA effect size of the significantly different bacterial taxa (left). The cladogram illustrates the phylogenetic relationships among the significantly different bacterial taxa. The dots in the center represent the OTUs at the phylum level, while the dots in the outer circle represent the OTUs at the species level. Coloring principles: species with no significant difference were uniformly colored yellow; species for biomarkers were colored according to the different groups, with red nodes indicating bacteria with important roles in the AMA group, and green nodes indicating bacteria with important roles in the normal group. The names of the species represented by a, b, c, d, and e in the figure are shown in the LDA score illustration on the right. Indicators were defined as those with LDA $>4$. The histogram shows the LDA scores computed for significantly abundant taxa between the normal and AMA groups. The histogram represents the most significantly abundant taxa between the two groups. AMA: bacterial groups in the AMA group; normal: bacterial groups in the normal group

ultrasonic guidance; the operating room was sterile and the surgical instruments underwent aseptic processing and packaging. Nine samples from chromosomally normal pregnancies were chosen randomly.

\section{$16 \mathrm{~S}$ rRNA gene sequencing}

Microbial DNA was extracted from AF samples using an E.Z.N.A. Stool DNA Kit (Omega Biotek, Norcross, GA, USA) according to the manufacturer's protocol. The 
Table 1 Clinical information of the newborns

\begin{tabular}{|c|c|c|c|c|c|c|c|c|}
\hline No. & Age & Apgar & Delivery modes & $\begin{array}{l}\text { Gestational } \\
\text { weeks }\end{array}$ & $\begin{array}{l}\text { W/L (birth) (kg/ } \\
\mathrm{cm})\end{array}$ & $\begin{array}{l}\text { W/L (90 days) } \\
(\mathrm{kg} / \mathrm{cm})\end{array}$ & Feeding & Allergy \\
\hline YSO1 & 29 & $10 / 10$ & Eutocia & $38^{+6}$ & $3.0 / 50$ & $7.0 / 61$ & PB & No \\
\hline YSO2 & 33 & $10 / 10$ & Cesarean & $37^{+5}$ & $3.4 / 49$ & $6.3 / 64$ & B & No \\
\hline YSO3 & 30 & $10 / 10$ & Eutocia & $38^{+3}$ & $2.9 / 50$ & $6.8 / 62$ & $\mathrm{~PB}$ & No \\
\hline YSO4 & 28 & $10 / 10$ & Cesarean & $38^{+5}$ & $3.4 / 50$ & $6 / 61.5$ & B & No \\
\hline YSO6 & 41 & $10 / 10$ & Cesarean & $39^{+2}$ & $3.4 / 50$ & $10 / 63$ & B & No \\
\hline YSO7 & 40 & $10 / 10$ & Eutocia & $39^{+0}$ & $3.5 / 50$ & $6.5 / 61$ & PB & No \\
\hline YS08 & 40 & $10 / 10$ & Eutocia & $37^{+5}$ & $3.3 / 48$ & $7.0 / 62$ & B & No \\
\hline YSO9 & 39 & $10 / 10$ & Eutocia & $39^{+5}$ & $4.1 / 52$ & $5.0 / 65$ & B & No \\
\hline YS10 & 40 & $10 / 10$ & Eutocia & $40^{+3}$ & $4.0 / 51$ & $9.0 / 65$ & B & No \\
\hline
\end{tabular}

Advanced maternal age was defined as age $>35$ years

$W$ weight; $L$ length; $P B$ partial breastfeeding; $B$ breastfeeding

16S rDNA V3-V4 region of the eukaryotic rRNA gene was amplified by PCR using the following thermal profile: $95{ }^{\circ} \mathrm{C}$ for $2 \mathrm{~min}$, followed by 27 cycles of $98{ }^{\circ} \mathrm{C}$ for $10 \mathrm{~s}, 62^{\circ} \mathrm{C}$ for $30 \mathrm{~s}$, and $68{ }^{\circ} \mathrm{C}$ for $30 \mathrm{~s}$, and a final extension at $68{ }^{\circ} \mathrm{C}$ for $10 \mathrm{~min}$. The primers used were 341-F: 5'-CCTACGGGNGGCWGCAG-3'and 806-R: 5'-GGA CTACHVGGGTATCTAAT- 3 ', where the barcode was an 8 bp sequence unique to each sample. The PCR amplifications were performed in triplicate using $50-\mu \mathrm{l}$ mixtures containing $5 \mu \mathrm{l}$ of $10 \times \mathrm{KOD}$ buffer, $5 \mu \mathrm{l}$ of $2.5 \mathrm{mM}$ dNTPs, $1.5 \mu \mathrm{l}$ of each primer $(5 \mu \mathrm{M}), 1 \mu \mathrm{l}$ of KOD polymerase, and $100 \mathrm{ng}$ of template DNA.

Amplicons were extracted from $2 \%$ agarose gels and purified using an AxyPrep DNA Gel Extraction Kit (Axygen Biosciences, Union City, CA, USA) according to the manufacturer's instructions, and quantified using a QuantiFluor-ST System (Promega, Madison, WI, USA). Purified amplicons were pooled in equimolar quantities and subjected to paired-end sequencing $(2 \times 250)$ on an Illumina HiSeq 2500 Platform (Illumina Inc., San Diego, CA, USA) according to standard protocols.

\section{Reanalysis of human AF raw data}

The wiff. MS data files from Liu et al. [11] were searched against the Swiss-Prot database (Homo sapiens, Acidobacteria, Actinobacteria, Bacteroidetes, Chloroflexi, Cyanobacteria, Firmicutes, Gammaproteobacteria, Patescibacteria, Planctomycetes, and Verrucomicrobia protein sequences, release 2020_06) [19] using MaxQuant software (version 1.3.0.5) [20]. False discovery rates (FDRs) were estimated using the targetdecoy strategy, and the FDR cut-offs were set to 0.01 for sites, peptides, and proteins. Enzyme specificity was considered to be full cleavage by trypsin, and two maximum missed cleavage sites were permitted. The minimum required peptide length was set to 7 residues.
Carbamidomethyl (C) and iTRAQ 8plex labels were set as fixed modifications. Variable modifications included oxidation (M) and acetylation (protein $\mathrm{N}$-term).

\section{Statistical analysis}

Bioinformatic analysis was performed using Omicsmart (http://www.omicsmart.com).

Abbreviation

AF: Amniotic fluid

\section{Supplementary Information}

The online version contains supplementary material available at https://doi. org/10.1186/s12967-021-02996-y.

Additional file 1. Human AF protein groups.

Additional file 2. Bacterial diversity indexes of the AF samples.

Additional file 3. LEfSe analysis of bacterial communities of the AF samples.

\section{Acknowledgements}

We are grateful to the affected individuals and the family for permitting us to publish this information, Wei Sun (Peking Union Medical College, China) for offering human AF proteomics database, and Guangzhou Genedenovo Biotechnology Co., Ltd., for providing technical support.

\section{Authors' contributions}

$\mathrm{PH}, \mathrm{YW}$ designed and performed the experiments; $C Y L$ and $L L$ were responsible for the clinical samples and data collection; ZFX, PH, YW, YWC analyzed the data; YW and DL drafted the manuscript. All authors read and approved the final manuscript.

\section{Funding}

This work was supported by Grants from the National Key R\&D Program of China (No. 2018YFC1002402), the National Natural Science Foundation of China (No. 81971398).

\section{Availability of data and materials}

The datasets used and/or analysed during the current study are available from the corresponding author on reasonable request. 


\section{Declarations}

Ethics approval and consent to participate

Samples of amniotic fluid were taken from each patient on the same day. All procedures performed in studies involving human participants were in accordance with the Declaration of Helsinki and the patients gave their written consent. The Research Ethics Committee of Nanjing Maternal and Child Health Hospital approved the study ([2019] KY-081).

\section{Consent for publication}

Not applicable.

\section{Competing interests}

The authors have declared that no competing interests exists.

\section{Author details}

'Department of Prenatal Diagnosis, Obstetrics and Gynecology Hospital Affiliated to Nanjing Medical University, Nanjing Maternity and Child Health Care Hospital, Nanjing 210004, China. ${ }^{2}$ Nanjing Jiangbei New Aera Biopharmaceutical Public Service, Platform No.3-1 Xinjinhu Road, Zhongdan Life Science and Ecology Industrial Park, Nanjing 210000, China.

Received: 13 April 2021 Accepted: 11 July 2021

Published online: 27 July 2021

\section{References}

1. Willyard C. Could baby's first bacteria take root before birth? Nature. 2018;553:264-6.

2. Lim ES, Rodriguez C, Holtz LR. Amniotic fluid from healthy term pregnancies does not harbor a detectable microbial community. Microbiome. 2018:6:87.

3. Payne MS, Keelan JA, Stinson LF. Re:"Amniotic fluid from healthy term pregnancies does not harbor a detectable microbial community." Microbiome. 2019;7:20.

4. Lim ES, Rodriguez C, Holtz LR. Reply Re: "Amniotic fluid from healthy term pregnancies does not harbor a detectable microbial community." Microbiome. 2019:7:21.

5. Cho C-KJ, Shan SJ, Winsor EJ, Diamandis EP. Proteomics analysis of human amniotic fluid. Mol Cell Proteom. 2007;6:1406-15.

6. Liu Y, Li X, Zhu B, Zhao H, Ai Q, Tong Y, et al. Midtrimester amniotic fluid from healthy pregnancies has no microorganisms using multiple methods of microbiologic inquiry. Am J Obstet Gynecol. 2020;223:248.

7. Blaser MJ, Devkota S, McCoy KD, Relman DA, Yassour M, Young VB. Lessons learned from the prenatal microbiome controversy. Microbiome. 2021;9:1-7.
8. Tsangaris GT, Kolialexi A, Karamessinis PM, Anagnostopoulos AK, Antsaklis A, Fountoulakis $\mathrm{M}$, et al. The normal human amniotic fluid supernatant proteome. Vivo. 2006;20:479-90.

9. Mishra A, Lai GC, Yao LJ, Aung TT, Shental N, Rotter-Maskowitz A, et al. Microbial exposure during early human development primes fetal immune cells. Cell. 2021;184:1-16.

10. Romero R, Dey SK, Fisher SJ. Preterm labor: one syndrome, many causes. Science. 2014;345:760-5.

11. Liu X, Song Y, Guo Z, Sun W, Liu J. A comprehensive profile and inter-individual variations analysis of the human normal amniotic fluid proteome. $J$ Proteom. 2019:192:1-9.

12. Heffner $\sqcup$ J. Advanced maternal age-how old is too old? N Engl J Med. 2004:351:1927-9.

13. Zhang $X$, Chen L, Wang $X$, Wang $X$, Jia M, Ni S, et al. Changes in maternal age and prevalence of congenital anomalies during the enactment of China's universal two-child policy (2013-2017) in Zhejiang Province, China: an observational study. PLoS Med. 2020;17:1-19.

14. Gomez-Lopez N, Romero R, Garcia-Flores V, Xu Y, Leng Y, Alhousseini A, et al. Amniotic fluid neutrophils can phagocytize bacteria: a mechanism for microbial killing in the amniotic cavity. Am J Reprod Immunol. 2017;78:139-48.

15. Collado MC, Rautava S, Aakko J, Isolauri E, Salminen S. Human gut colonisation may be initiated in utero by distinct microbial communities in the placenta and amniotic fluid. Sci Rep. 2016;6:23129.

16. Jiménez E, Marín ML, Martín R, Odriozola JM, Olivares M, Xaus J, et al. Is meconium from healthy newborns actually sterile? Res Microbiol. 2008:159:187-93.

17. Dennis Lo YM, Corbetta N, Chamberlain PF, Rai V, Sargent IL, Redman CWG, et al. Presence of fetal DNA in maternal plasma and serum. Lancet. 1997;350:485-7.

18. Vernon SD, Shukla SK, Conradt J, Unger ER, Reeves WC. Analysis of 165 rRNA gene sequences and circulating cell-free DNA fromplasma of chronic fatigue syndrome and non-fatigued subjects. BMC Microbiol. 2002;2:1-6.

19. Magrane M, UniProt Consortium. UniProt Knowledgebase: a hub of integrated protein data. Database. 2011;2011:bar009.

20. Cox J, Mann M. MaxQuant enables high peptide identification rates, individualized p.p.b.-range mass accuracies and proteome-wide protein quantification. Nat Biotechnol. 2008;26:1367-72.

\section{Publisher's Note}

Springer Nature remains neutral with regard to jurisdictional claims in published maps and institutional affiliations.
Ready to submit your research? Choose BMC and benefit from:

- fast, convenient online submission

- thorough peer review by experienced researchers in your field

- rapid publication on acceptance

- support for research data, including large and complex data types

- gold Open Access which fosters wider collaboration and increased citations

- maximum visibility for your research: over 100M website views per year

At $B M C$, research is always in progress.

Learn more biomedcentral.com/submissions 